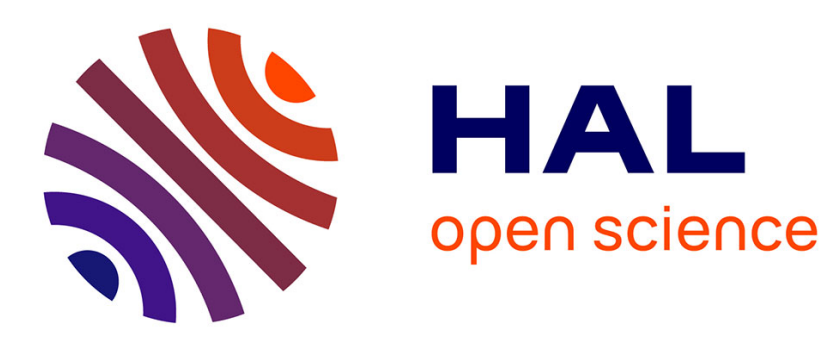

\title{
A direct link between microstructure and acoustical macro-behavior of real double porosity foams
}

\author{
F. Chevillotte, Camille Perrot, E. Guillon
}

\section{To cite this version:}

F. Chevillotte, Camille Perrot, E. Guillon. A direct link between microstructure and acoustical macrobehavior of real double porosity foams. Journal of the Acoustical Society of America, 2013, 134 (6), pp.4681-4690. 10.1121/1.4824842 . hal-00817040v2

\section{HAL Id: hal-00817040 \\ https://hal.science/hal-00817040v2}

Submitted on 9 Jun 2015

HAL is a multi-disciplinary open access archive for the deposit and dissemination of scientific research documents, whether they are published or not. The documents may come from teaching and research institutions in France or abroad, or from public or private research centers.
L'archive ouverte pluridisciplinaire HAL, est destinée au dépôt et à la diffusion de documents scientifiques de niveau recherche, publiés ou non, émanant des établissements d'enseignement et de recherche français ou étrangers, des laboratoires publics ou privés. 


\title{
A direct link between microstructure and acoustical macro-behavior of real double porosity foams
}

\author{
Fabien Chevillotte ${ }^{\text {a) }}$ \\ Matelys-Acoustique et Vibrations, 1 rue Baumer, F69120 Vaulx-en-Velin, France \\ Camille Perrot \\ Université Paris-Est, Laboratoire Modélisation et Simulation Multi Echelle, MSME UMR 8208 CNRS, \\ 5 bd Descartes, 77454 Marne-la-Vallée, France
}

Emmanuel Guillon

Lafarge Research Centre, Saint Quentin Fallavier, France

(Received 27 September 2012; revised 11 April 2013; accepted 22 April 2013)

\begin{abstract}
The acoustical macro-behavior of mineral open-cell foam samples is modeled from microstructure morphology using a three-dimensional idealized periodic unit-cell (3D-PUC). The 3D-PUC is based on a regular arrangement of spheres allowed to interpenetrate during the foaming process. Identification and sizing of the 3D-PUC is made from x-ray computed microtomography and manufacturing process information. In addition, the 3D-PUC used allows to account for two scales of porosity: The interconnected network of bubbles (meso-porosity) and the inter-crystalline porosity of a gypsum matrix (micro-porosity). Transport properties of the micro- and the meso-scales are calculated from first principles, and a hybrid micro-macro method is used to determine the frequency-dependent visco-thermal dissipation properties. Olny and Boutin found that the double porosity theory provides the visco-thermal coupling between the meso- and micro-scales [J. Acoust. Soc. Am. 114, 73-89 (2003)]. Finally, the results are successfully compared with experiments for two different mineral foam samples. The main originality of this work is to maintain a direct link between the microstructure morphology and the acoustical macro-behavior all along the multi-scale modeling process, without any adjusted parameter.
\end{abstract}

(C) 2013 Acoustical Society of America. [http://dx.doi.org/10.1121/1.4824842]

PACS number(s): 43.50.Gf, 43.55.Ev, 43.20.Gp [KVH]

Pages: 4681-4690

\section{INTRODUCTION}

Linking the microstructure of a material to its macroscopic properties is of great interest for understanding the functional properties of a material and for its optimization. During the last years, the micro-macro methods have been the subject of numerous investigations and are currently the subject of much attention. ${ }^{1-5}$ Nowadays, three-dimensional x-ray computed microtomography $(\mu \mathrm{CT})$ and numerical modeling tools enable solving various transport problems through porous media. Does the combination of these techniques allow to understand the material behavior and optimize the microstructure to enhance the performances? This might be the main question leading studies on relationships between structure and properties of porous media.

Gasser et al. ${ }^{1}$ treated the three-dimensional case of facecentered nickel hollow spheres packings with a view of absorbing sound inside the turboengines of aircrafts. The geometric simplicity of the structure directly provides the local geometry parameters for the numerical models. Special care was given to properly model solder joints.

Perrot et $a l^{2}{ }^{2}$ presented a hexagonal lattice of solid fibers with circular cross-section shape for the dynamic viscous permeability of an open cell aluminum foam. They used

\footnotetext{
a) Author to whom correspondence should be addressed. Electronic mail: fabien.chevillotte@matelys.com
}

X-ray computed microtomography to characterize the purely geometrical macroscopic parameters with porosity and thermal length as input parameters of the two-dimensional model. The orientation of the solid fibers controlled the static viscous permeability that was underestimated by the simple two-dimensional model.

Lee et $\mathrm{ll}^{3}{ }^{3}$ paid attention to the three-dimensional hexagonal closed pack structure that has not appeared in the acoustic literature previously and showed that multi-periodic composite structures, defined as periodically layered media wherein each layer is composed of periodic unit-cell, could lead to frequency stop-bands.

Venegas and Umnova ${ }^{4}$ modeled the acoustic properties of simple [(a) an irregular packing of lead shots] and double porosity unconsolidated granular materials with low [(b) expanded perlite] and high [(c) activated carbon] permeability contrasts. (a) The analytical model developed by Boutin and Geindreau ${ }^{6}$ from the standpoint of selfconsistent and periodic structures homogenizations was found to be fairly applicable to the irregular packing of lead shots. (b) SEM images were used to characterize the cell size of the microporous network when dealing with the sample of expanded perlite, whereas the diameter of the perforations interconnecting cells was somewhat arbitrarily set to half of the smallest face of the truncated octahedron. This describes all the relevant parameters of the microporous domain to numerically determine the corresponding 
transport parameters. The Boutin and Geindreau model was used to describe the mesoporous domain, taking half of the average between the upper and lower limit of the $80 \%$ band grain size as an input parameter. The coupling between the micro- and meso-porous domains was provided by Olny and Boutin double porosity theory ${ }^{7}$ with an analytical calculation of the pressure diffusion function. A reasonably good agreement was found between this hybrid modeling strategy and the experimental data. A better agreement was obtained by fitting the analytical double porosity model to the data with the microporous domain simply replaced by circular cylinders. (c) The analytical double porosity model was also used for the activated carbon samples. The mesopore radius was obtained from optical granulometry, whereas the micropore one was derived from a fitting routine procedure.

Perrot et $a l .{ }^{5}$ numerically determined the high-frequency transport parameters and the acoustic properties of polyurethane foam samples, including polydispersity, anisotropy, and membrane effects. The porosity and permeability were used as input data for the scaling of a three-dimensional open cell.

No direct link between microstructure and acoustical macro-behavior was therefore given in all of these research works for disordered real porous materials.

The major purpose of this work is to make a detailed study of two different gypsum foams that consists of three steps. First, the gypsum foam microstructure is characterized by using a morphology inspired representative elementary volume combined with microtomography. Second, the gypsum foam transport properties are numerically computed by solving the local homogenized equations in the (micro- and) meso-porous structures characterized in the first step. In addition, the acoustical macro-behavior is analytically determined from these computations. Third, pore scale predictions are compared to experimental values.

Foamed gypsum structures are porous materials composed of interpenetrating bubbles that connect through a (micro-porous) solid matrix to form three-dimensional networks. Gypsum foams may find large applications for many industrial applications including building materials because of their mechanical, thermal, fire resistant, and acoustical properties. Owing in the progress in manufacturing methods, gypsum foams can now be produced with controlled microstructure, ${ }^{8}$ which might be optimized for acoustic purposes. Specifically for noise control engineering, manufacturing methods can be used for the generation of porous gypsum materials with essentially two types of microstructures that facilitate sound absorption and transmission loss (for further details, see Sec. II).

There are mainly two types of methods for modeling the acoustical macro-behavior of a porous media from its microstructure, Fig. 1. On the one hand, a direct dynamic method can be used for calculating the frequency-dependent transport parameters at each frequency of interest ${ }^{9}$ on a $\mu \mathrm{CT}$ of a real porous sample (Fig. 1, left). The so-called direct method has the advantage to preserve a realistic representation of the microstructure, but it is time and memory consuming. More importantly, it does not explain how the microstructural features lead the macroscopical results. Thus these limits suggest that this last strategy is not the most suitable for optimization purposes. On the other hand, a hybrid method can be used for determining the dynamic behavior from asymptotic analysis $^{1-5}$ on an idealized periodic unit cell (PUC) (Fig. 1, right). This type of method relies on approximate but robust semi-phenomenological models, ${ }^{10-13}$ the macroscopic input parameters being computed from a geometrical model of the microstructure. This hybrid method is fast and provides the macroscopic parameters that have physical meaning and that can be very helpful for apprehending the micro-physical basis behind the acoustical macro-behavior. ${ }^{14}$ This approach is thus more adapted for optimization; however, the identification of the PUC is not trivial. Indeed one can find a PUC representative of the physical parameters at the engineering scale that does not correspond to the real porous media. That is, there is uniqueness of the micro-macro solution, contrary to the macro-micro one. A main difficulty relies on getting an agreement between the input parameters of the microstructural model and the measured parameters from the real microstructure. Thus from this point of view, the main issue is to identify the shape and characteristic sizes of the PUC.

The paper is organized as follows. The identification procedure of the representative periodic unit cell is described in Sec. II. Then we briefly recall the basic equations used to calculate the acoustical macro-behavior in Sec. III. This theoretical summary deals with the hybrid micro-macro
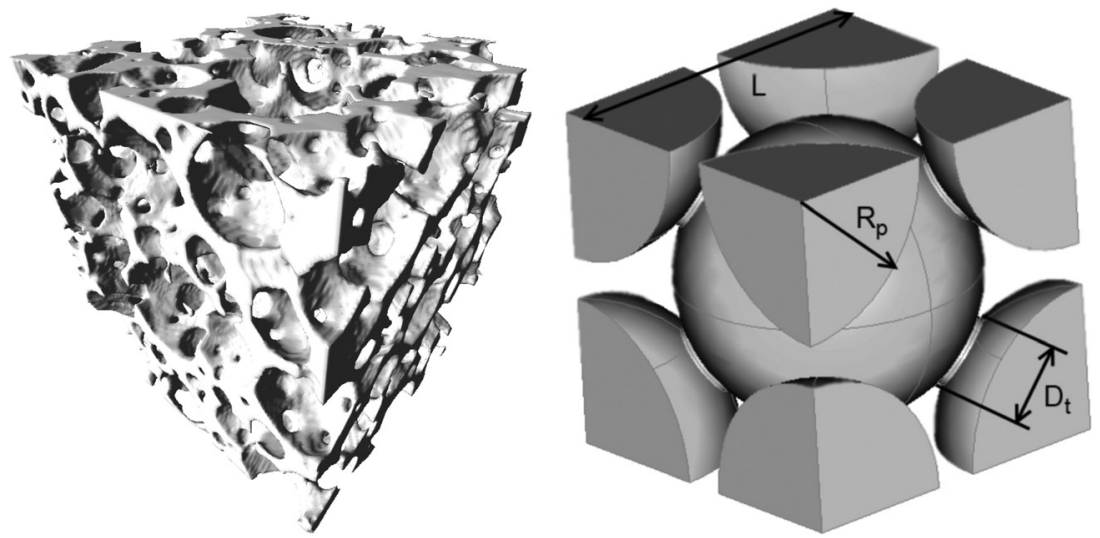

FIG. 1. Example of real computed microtomography scan (left) versus idealized periodic unit cell (right). 


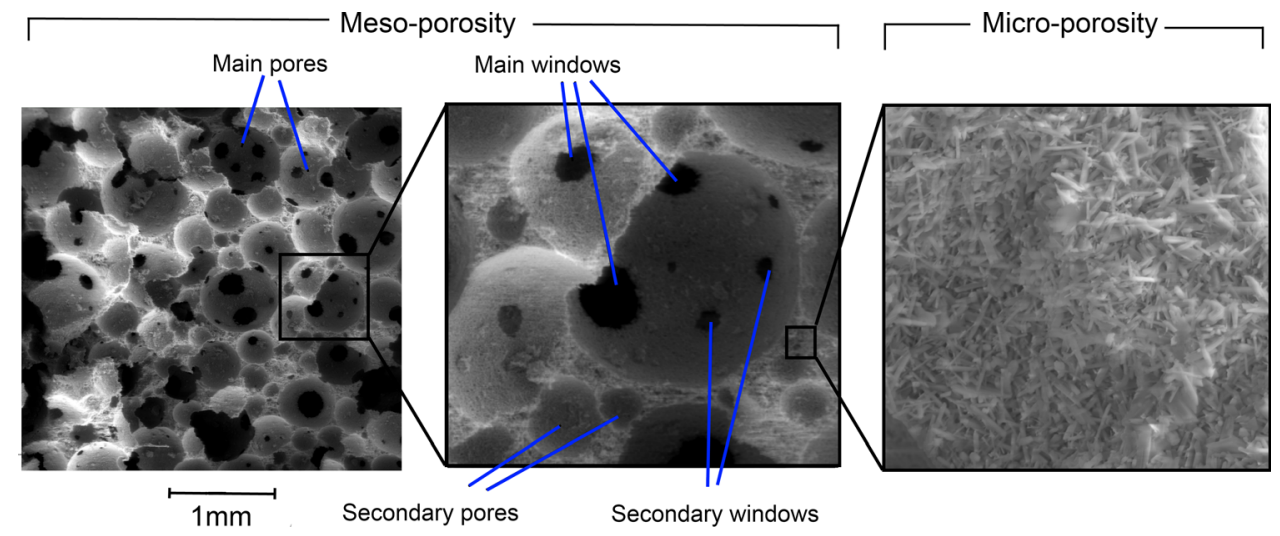

FIG. 2. (Color online) Microstructure of porous gypsum at meso- and micro-scale.

method, the single porosity model, and the double porosity model. Finally, we present and discuss the results of our study for two materials in Sec. IV.

\section{MICROSTRUCTURE CHARACTERIZATION}

Looking at the microstructure (Figs. 2 and 3), the first step is to choose a periodic unit cell (PUC) and size it. The proposal and the sizing of the PUC will be presented in this section.

\section{A. 3D-PUC proposal}

Gypsum foams considered in this study result from a foaming process. There are numerous foaming processes, but the principle is always to make growing gas bubbles within a liquid matrix. In three dimensions, the most simple idealized cell is a regular positioning of spheres with identical sizes. Using a model with an increasing complexity, this type of regular arrangement is first chosen. If it shows limitations, a complexification will be considered. According to the geometric modeling adopted, growing bubbles will tend to interconnect pores and create a network of open pores. The main question remains as to the following type of arrangement (simple cubic, bodycentered cubic, face-centered-cubic). The physics of foams suggests that the body-centered cubic (BCC) arrangement is the most appropriate because it tends to the Kelvin cell (tetrakaidecahedron) when pores are growing. The Kelvin

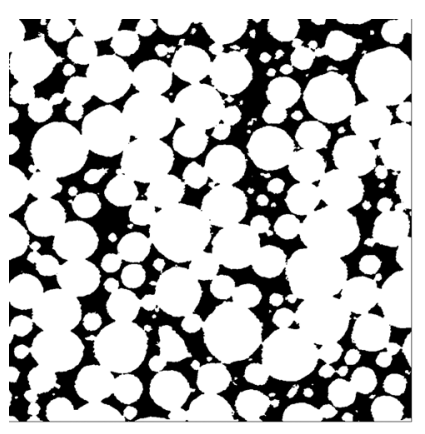

a)

FIG. 3. Examples of binarized slice tomography of gypsum foams. (a) Material A, (b) material B. cell is well known for modeling high porosity foams as polyurethane or polymer foams. ${ }^{15}$ The BCC cell is illustrated in Fig. 1 (right).

\section{B. Sizing the PUC}

\section{Porosity}

A plate of gypsum has a micro-porous matrix. During the foaming process, a meso-porous network is created. We distinguish the micro-porous matrix (Fig. 2, right), and the meso-porous network (Fig. 2, left), called bubble porosity. The x-ray tomography does not have enough resolution to identify the micro-porous matrix of gypsum but allows access to the porosity of bubbles. The voxel resolution is $5 \mu \mathrm{m}$. The porosity of bubble (meso) network $\phi_{\text {meso }}$ is directly calculated from each extracted slice and averaged over the total volume. A limitation of this method is that there is no distinction between the open and the closed porosity. The closed porosity is considered negligible compared with the open one for the studied materials. In addition, as the gypsum matrix is porous, the pores can be considered as interconnected. Examples of binarized slices are shown in Fig. 3.

\section{Pore size}

The pore size distribution is extracted from particle size analysis. The principle of the algorithm is summarized in the following text. First a binarization is performed. The thresholding is performed from the analysis of the histogram of the gray levels after applying a noise correction filter and increasing the contrast. It is manually adjusted to ensure that the number of artifacts is limited. Starting from the binarized three-dimensional (3D) image, an erosion operation, as defined in mathematical morphology, is iteratively carried out until finding the centers of the pores. The smallest radius of each pore is given by the number of iterations and the size of the voxels. Then starting from these centers of pores, a dilation operation is applied for the number of iterations previously determined while growing a spherical pore. Finally, the pores are enlarged to complete the voxels of pore space initially binarized. The pore volumes are approximated by spheres with an equivalent radius. This equivalent radius relatively well matches to the smallest radius because the real 
pore shapes are close to spheres. Some voxels belong to several pores. The voxels belonging to two pores constitute the interconnection volume. ${ }^{16,17}$

The median diameter, $D_{p} 50$ is used instead of the arithmetic average because the median mitigates the disturbing influence of the extremal values (low number of very small or very large pores). The pore size diameters must be weighted by the volumes to give a representative pore size distribution. A good way to check the representativeness of the pore size distribution is to compare with measurements on 2D micrographs.

\section{Throat size}

The throat size is a main parameter leading the acoustical macro-behavior because it controls the static air flow resistivity and the viscous characteristic length. Contrary to the mean pore size diameter, the automatic estimation of the throat size is not that easy from $3 \mathrm{D}$ tomography postprocessing. Because the stack of binarized slices is available, it is possible to manually scroll through the slices to measure the throat size by looking for the maximum diameter of each throat. This approach will be called the manual method.

Another way to estimate the throat size is to consider the interconnection volume and reach it by an ellipsoid as depicted in Fig. 4. The previously described algorithm also provides interconnection volumes $V_{i}$. Then the interconnection volumes can be approximated by ellipsoids with axis $\left(D_{t}, D_{t}, D_{t} / r\right)$. The throat diameter $D_{t}$ writes

$$
D_{t}=2 \sqrt[3]{\frac{3}{4 \pi} V_{i} r} .
$$

This method will be called the automated one.

The first method is somewhat subjective because it depends on the operator and the second one needs an estimation of the axis ratio $r$. The manual throat size determination can be used to estimate this ratio $r$. Then, Eq. (1) can be applied on all interconnection volumes. This second method provides a better statistical representativeness and allows checking the relevance of the manual throat size distribution

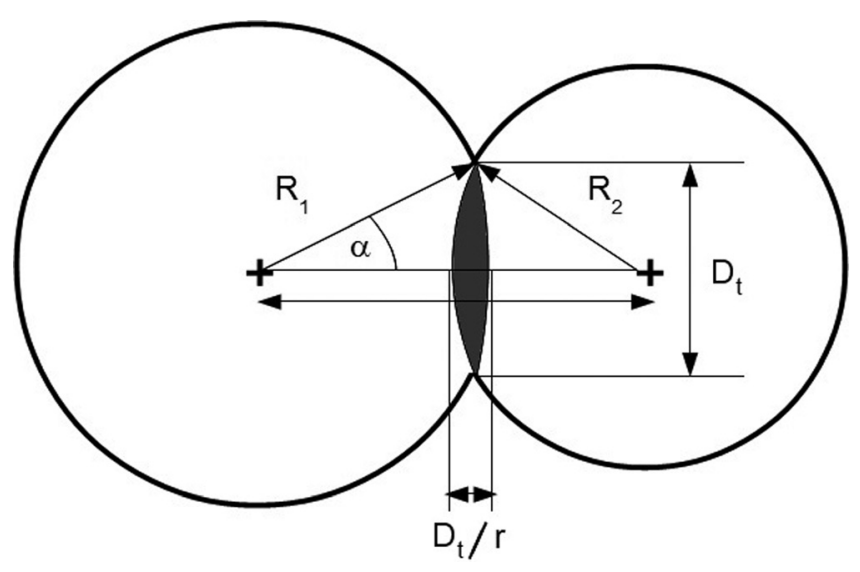

FIG. 4. Scheme of interconnection. (shape, spreading). Both of these methods will be used in this work.

\section{ACOUSTICAL MACRO-BEHAVIOR: THEORETICAL SUMMARY}

The macroscopic description of long-wavelength acoustic propagation and dissipation phenomena through an interconnected fluid filled rigid porous media may be modeled as an equivalent fluid characterized by two complex frequency-dependent functions: The dynamic density $\tilde{\rho}_{e q}(\omega)$, which takes into account the visco-inertial interaction between the motionless frame and the saturating fluid, and the dynamic bulk modulus $\tilde{K}_{e q}(\omega)$, which takes into account the thermal interaction. The symbol " " defines complex quantities.

\section{A. Hybrid micro-macro method}

Contrary to the direct approach, which solves the linearized Navier-Stokes and the heat equations in harmonic regime, the hybrid method relies on approximate but robust semi-phenomenological models [Johnson-Champoux-Allard (JCA), Johnson-Champoux-Allard-Lafarge (JCAL)]. ${ }^{10-13}$ These models are very attractive because they avoid computing the solution of the full frequency range values of the effective density/bulk modulus. The principle is to solve the local equations governing the asymptotic frequency-dependent visco-thermal dissipation phenomena on the proposed PUC. All the macroscopic parameters of interest can be determined from only three asymptotic calculations:

(1) The open porosity $\phi$ is defined as the fraction of the interconnected pore fluid volume to the total bulk volume of the porous aggregate. A second parameter that is widely used to characterize the macroscopic geometry of porous media is the hydraulic radius, defined as twice the ratio of the total pore volume to its surface area. This characteristic length may also be referred to as the thermal characteristic length $\Lambda^{\prime}$ in the context of sound absorbing materials. ${ }^{12}$ Both of these parameters are estimated by direct spatial integration on the volume and surface elements of the $3 \mathrm{D}$ reconstructed periodic unit cell.

(2) The static airflow resistivity $\sigma$ (or static viscous permeability $k_{0}=\eta / \sigma$, where $\eta$ is the dynamic fluid viscosity) is computed from the steady Stokes problem ${ }^{18}$ also known as the low Reynolds number viscous flow equations (the symmetry properties of the microstructure under consideration implies that the second order permeability tensor is isotropic and reduced to a scalar $k_{0}$ ). The static viscous tortuosity $\alpha_{0}$, another transport parameter significant of the viscous flow, might also be computed from the same boundary value problem. It was, however, not considered here because direct experimental evaluations of this parameter is still an open problem.

(3) The viscous characteristic length $\Lambda$ and the high frequency limit of the tortuosity $\alpha_{\infty}$ are calculated using a 
perfect (non viscous) incompressible fluid which formally behaves according to the electric conduction. ${ }^{19}$ They are determined from solutions of the Laplace's equation. $\Lambda$ should be interpreted as a weighted volumeto-surface ratio for the throat region. One way to interpret the high frequency limit of the tortuosity is to see that the effective traveled distance for the wave between two points is increased by $\sqrt{\alpha_{\infty}}$ due to the tortuosity of the path.

(4) The static thermal permeability $k_{0}^{\prime}$, also known as the inverse of the trapping constant $\Gamma$ in the context of physical chemistry - where surface exchanges play an important role-was computed by means of thermal conduction problem where the solid skeleton is considered as a thermostat. There is a formal analogy with diffusion-controlled reactions. ${ }^{20}$ An additional macroscopic parameter, the static thermal tortuosity $\alpha_{0}^{\prime}$, might be derived from the same boundary value problem.

\section{B. Single porosity model}

The two complex frequency-dependent functions [the dynamic density $\tilde{\rho}_{e q}(\omega)$ and the dynamic bulk modulus $\left.\tilde{K}_{e q}(\omega)\right]$ are then analytically derived from computed macroscopic parameters, on the $3 \mathrm{D}$ periodic unit cell, using approximate but robust JCAL semi-phenomenological model. ${ }^{10-13}$ It might be useful to recall that the main ingredients to build this model are to account for the causality principle and therefore for the Kramers-Kronig relations between real and imaginary parts of the frequencydependent dynamic density and dynamic bulk modulus. The considered macroscopic parameters, in these models, are those that correctly match the frequency dependence of the leading terms on the exact results for the high- and low- frequency behaviors. The models for the effective response functions were shown to agree with those calculated directly or independently measured. An important feature of this theory is that all of the parameters in the models can be independently calculated, most of them being in addition directly measurable in nonacoustical experimental situations. The dynamic density and dynamic bulk modulus have a two-asymptotic-state behavior. The visco-inertial dissipations are governed by viscous forces at low frequencies and by inertial forces at high frequencies. These two behaviors are separated by a characteristic angular frequency that can be approximated by the analytical value associated to cylinders of circular cross-section shape and given by $\omega_{v}=\nu \phi$ / $\left(k_{O} \alpha_{\infty}\right)$ ( $\nu$ is the kinetic viscosity). Similarly, the thermal dissipations are considered isothermal at low frequencies and adiabatic at high frequencies. The regime transition is characterized by a thermal characteristic angular frequency for which the analytical value is $\omega_{t}=\nu^{\prime} \phi / k_{O}^{\prime}\left(\nu^{\prime}=\nu / \operatorname{Pr}\right.$, Pr being the Prandtl number).

\section{Double porosity model}

The microstructure, illustrated in Fig. 2, shows a mesoscopic network and a micro-porous matrix. The complex frequency-dependent functions of the mesoscopic scale $\left[\tilde{\rho}_{\text {eq,meso }}(\omega), \tilde{K}_{\text {eq,meso }}(\omega)\right]$ and the microscopic scale $\left[\tilde{\rho}_{\text {eq, micro }}(\omega), \tilde{K}_{\text {eq, micro }}(\omega)\right]$ can be computed by a micromacro method as described Sec. III A and coupled by a double porosity model. ${ }^{7}$ The equivalent fluid properties of the double porosity media $\left[\tilde{\rho}_{e q, d p}(\omega), \tilde{K}_{e q, d p}(\omega)\right]$ write

$$
\tilde{\rho}_{e q, d p}(\omega)=\frac{1}{\frac{\left(1-\phi_{\text {meso }}\right)}{\tilde{\rho}_{\text {eq, micro }}(\omega)}+\frac{1}{\tilde{\rho}_{\text {eq, meso }}(\omega)}}
$$

and

$$
\tilde{K}_{e q, d p}(\omega)=\frac{1}{\frac{\left(1-\phi_{\text {meso }}\right) F_{d}}{\tilde{K}_{e q, \text { micro }}(\omega)}+\frac{1}{\tilde{K}_{\text {eq, meso }}(\omega)}},
$$

with $F_{d}$ the dynamic diffusion function. Note that the diffusion is neglected in this work since the characteristic pressure diffusion frequency $f_{d}$ is approximately $12000 \mathrm{~Hz}$ for the two considered materials, which is higher than the frequency range of interest $(0-5000 \mathrm{~Hz})$

$$
f_{d}=\frac{1}{2 \pi} \frac{\left(1-\phi_{\text {meso }}\right) P_{0}}{\phi_{\text {micro }} \sigma_{\text {micro }} D(0)},
$$

with $P_{0}$ the atmospheric pressure and $D(0)$ is a transport parameter similar to a static permeability that depends on the geometry. ${ }^{7}$

Note that diffusion could be taken into account by calculating the parameter $D(0)$, which reduces to a calculation of permeability in the complementary cell of the PUC used for modeling the meso-porous bubbles network (Fig. 1, right). This calculation could be achieved according to the steady Stokes problem. ${ }^{18}$

The porosity $\phi_{d p}$ and the permeability $k_{0, d p}$ of the double porosity media are given by

$$
\phi_{d p}=\phi_{\text {meso }}+\left(1-\phi_{\text {meso }}\right) \phi_{\text {micro }}
$$

and

$$
k_{0, d p}=k_{0, \text { meso }}+\left(1-\phi_{\text {meso }}\right) k_{0, \text { micro }} .
$$

\section{RESULTS AND DISCUSSION}

\section{A. Microstructure}

Two mineral foam samples corresponding to different manufacturing processes were investigated. The porosity of the mesoscopic network, the pore size distribution and the throat size distribution have been measured according to Sec. II B. The volumes extracted by microtomography are, respectively, $900 \times 900 \times 900$ and $700 \times 700 \times 700$ voxels with a resolution of $5 \mu \mathrm{m}$. The bubble porosity was examined, and it was found that the first material appeared to be homogeneous $\left(\phi_{\text {meso }}=0.70 \pm 0.02\right)$, whereas the second one showed a very slight graded porosity $\left(\phi_{\text {meso }}=0.55 \pm 0.03\right)$. This was seen on the evolution of the porosity, extracted on 2D slices, when plotted as a function of the depth. Respectively, 6602 and 11676 pores have been detected in the acquired volume for materials A and B. And around 200 throat sizes have been 
manually measured on the stack of binarized slices for both of the experimental samples. Estimation of throat sizes from interconnection volumes have been carried out with an ellipsoid axis ratio $r=15$ for each of the experimental samples under study. Corresponding pore size and throat size distributions are shown in Figs. 5 and 6. The median pore size diameters $D_{p} 50$ are $460 \pm 140$ and $300 \pm 70 \mu \mathrm{m}$, respectively, for materials $\mathrm{A}$ and $\mathrm{B}$. The mean throat size diameters $D_{t}$ are, respectively, $110 \pm 40$ and $70 \pm 30 \mu \mathrm{m}$. Throat size distributions, obtained by the two methods described in Sec. II B 3, give comparable results. The comparison between the manual cumulative distribution and the automated one suggests that the manual measurement method was appropriate to throat size determination.

\section{B. Acoustical macro-behavior}

The two materials have been initially characterized as homogeneous single porosity materials. The porosity $\phi$ was measured from the perfect gas law properties using the method described by Beranek. ${ }^{21}$ The static airflow resisitivity $\sigma$ (and the static viscous permeability $k_{0}$ ) have been measured according to standard ISO 9053. The dynamic density and the dynamic bulk modulus are measured from impedance tube technique and the viscous $\Lambda$ and the thermal $\Lambda^{\prime}$ characteristic lengths, the high frequency limit tortuosity $\alpha_{\infty}$ and the static thermal permeability $k_{0}^{\prime}$ are derived from these two dynamic quantities by analytical determinations. $^{22,23}$ The analytical determination of the four last parameters is based on a single porosity behavior. Because the characteristic size of the micro-scale (porous matrix) is on the order of magnitude of micrometer, its airflow resistivity is very high and its diffusion frequency $f_{d}$ is also relatively high $(\approx 12000 \mathrm{~Hz})$. As a first approximation, this double porosity material can therefore be considered as a single porosity one with equivalent macroscopic parameters. All results are given in Tables I and II for materials $\mathrm{A}$ and $\mathrm{B}$, respectively. The experimental values and uncertainties have been determined from three different samples.
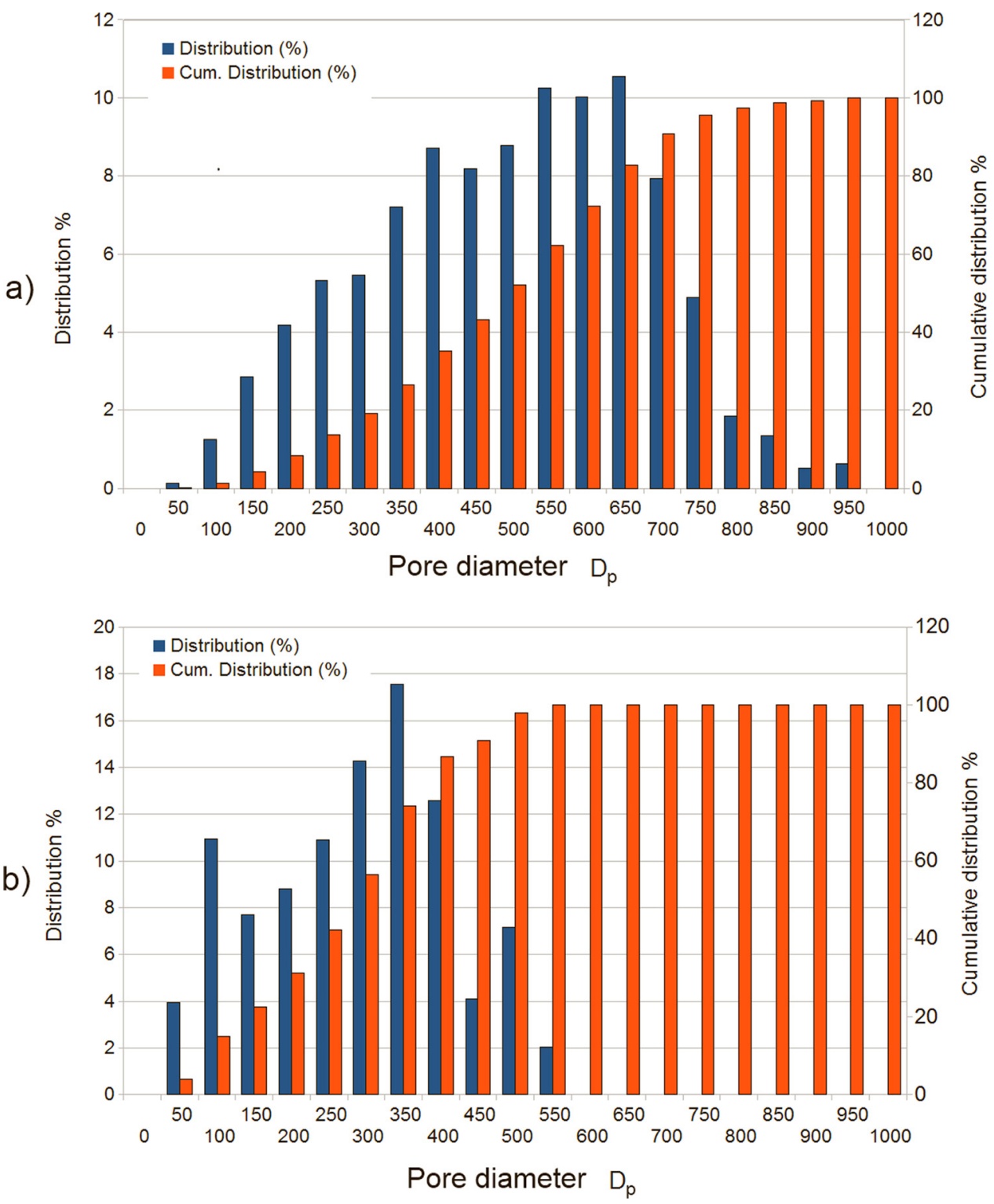

FIG. 5. (Color online) Pore size distribution: (a) Material A (top), (b) material B (bottom). 


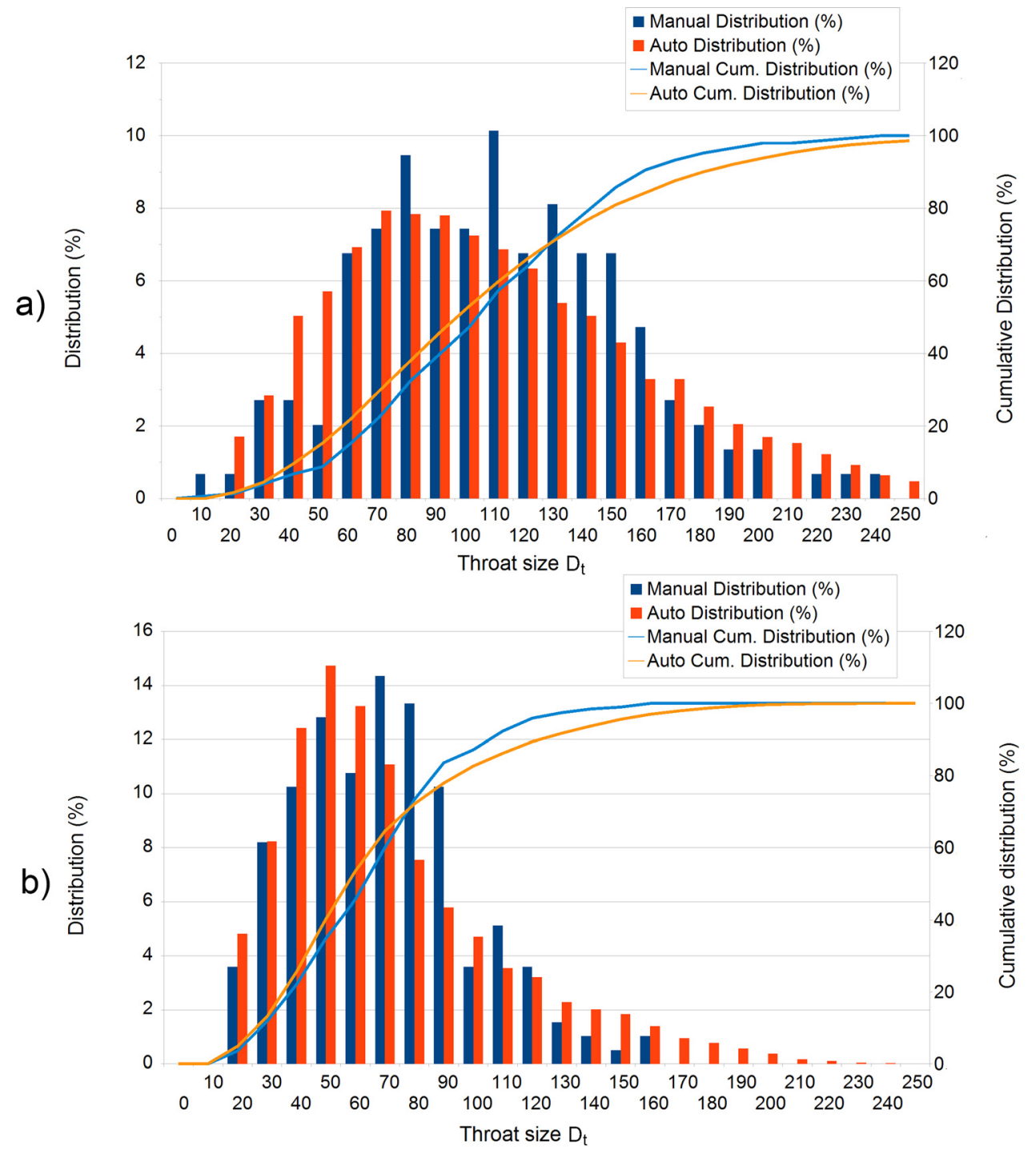

FIG. 6. (Color online) Throat size distribution: (a) Material A (top), (b) material B (bottom).

First we examined the single porosity model including only mesoscopic scale. The simulated single porosity parameters are deduced from the hybrid method of Sec. III A, where only the meso-porosity is considered in the PUC model. The simulated airflow resistivities are about 98100 and $383000 \mathrm{~N} \mathrm{~s} \mathrm{~m}^{-4}$, respectively, for materials A and B. These values are in good agreement with the results obtained from direct measurements performed on the real mineral foam samples. Simulated porosities are underestimated because only the meso-porous bubbles network is taken into account. The high frequency limits of tortuosities are in good compliance with measurements. Unfortunately, simulated viscous and thermal characteristic length are overestimated using this first geometrical model.

However, the micro-porosity of the gypsum matrix $\phi_{\text {micro }}$ is given by the mixing ratio. Its order of magnitude is

TABLE I. Material A: Characterized and simulated macroscopic parameters.

\begin{tabular}{lcccccc}
\hline \hline Cell & $\phi$ & $\sigma$ & $\Lambda^{\prime}$ & $\Lambda$ & $\alpha_{\infty}$ & $k_{0}$ \\
\hline Units & & $\mathrm{N} \mathrm{s} \mathrm{m}^{-4}$ & $\mu \mathrm{m}$ & $\mu \mathrm{m}$ & & $10^{-10} \mathrm{~m}^{2}$ \\
\hline Micro-porous matrix & 0.61 & $94810^{6}$ & 1.59 & 0.83 & 1.48 & 0.00178 \\
Single porosity (meso-scale) & 0.70 & 98100 & 168 & 62 & 2.16 & 1.88 \\
Single porosity_two throat sizes & 0.70 & 98100 & 168 & 42 & 2.16 & 1.88 \\
Double porosity & 0.88 & 96881 & 95 & 37 & 2.16 & 25 \\
Double porosity—two throat sizes & 0.88 & 96881 & 95 & 28 & 2.16 & 1.90 \\
Exp. & 0.86 & 80400 & 116 & 23 & 2.04 & 2.90 \\
& $(0.01)$ & $(6700)$ & $(17)$ & $(6)$ & $(0.38)$ & $(0.18)$ \\
\hline \hline
\end{tabular}


TABLE II. Material B: Characterized and simulated macroscopic parameters.

\begin{tabular}{|c|c|c|c|c|c|c|c|}
\hline Cell & $\phi$ & $\sigma$ & $\Lambda^{\prime}$ & $\Lambda$ & $\alpha_{\infty}$ & $k_{0}$ & $k_{0}^{\prime}$ \\
\hline Units & & $\mathrm{Ns} \mathrm{m}^{-4}$ & $\mu \mathrm{m}$ & $\mu \mathrm{m}$ & & $10^{-10} \mathrm{~m}^{2}$ & $10^{-10} \mathrm{~m}^{2}$ \\
\hline Micro-porous matrix & 0.61 & $94810^{6}$ & 1.59 & 0.83 & 1.48 & 0.00178 & 0.000194 \\
\hline Single porosity (meso-scale) & 0.55 & 383000 & 104 & 39 & 2.55 & 0.480 & 8 \\
\hline Double porosity & 0.82 & 379400 & 41 & 16 & 2.55 & 0.485 & 9 \\
\hline Exp. & $\begin{array}{c}0.85 \\
(0.01)\end{array}$ & $\begin{array}{l}402400 \\
(13100)\end{array}$ & $\begin{array}{c}595 \\
(688)\end{array}$ & $\begin{array}{c}4 \\
(0)\end{array}$ & $\begin{array}{c}1.81 \\
(0.11)\end{array}$ & $\begin{array}{l}0.457 \\
(0.18)\end{array}$ & $\begin{array}{c}48 \\
(16)\end{array}$ \\
\hline
\end{tabular}

0.6. The gypsum matrix porosity is deduced from the measurement of gypsum matrix mass density $\rho$ and the gypsum mass density in its crystalline form $\rho_{\text {crystal }}$,

$$
\phi_{\text {micro }}=1-\frac{\rho}{\rho_{\text {crystal }}},
$$

where the gypsum mass density in its crystalline form is given by $\rho_{\text {crystal }}=2300 \mathrm{~kg} \mathrm{~m}^{-3}$. ${ }^{24}$ This micro-porous matrix can be considered by using a double porosity model as described in Sec. III C. The dynamic functions of the microporous matrix have been simulated using a simple micromacro (cf. Sec. III A) model based on a pack of fibers with fiber diameters $d$ in the order of the micron and submicrometric throat sizes $w$ (see Fig. 7). The use of this simplified $2 \mathrm{D}$ cell is justified by the small size of throats involving that the acoustic behavior is mainly controlled by the airflow resistivity. This microstructure parameters $d$ and $w$ have been evaluated from micrographs (cf. Fig. 2). The equivalent fluid properties of the double porosity model can be reevaluated according to Eqs. (2) and (3).

The porosity and the static viscous permeability can thus be calculated with Eqs. (5) and (6). The four other macroscopic parameters are estimated from dynamic quantities, $\tilde{\rho}_{e q, d p}(\omega)$ and $\tilde{K}_{e q, d p}(\omega)$, in the same way as experimental values. ${ }^{22,23}$ This "numerical characterization" allows to compare macroscopic parameters of the different models in Tables I and II. This unit cell is called "double porosity cell." The porosities are now well evaluated. The static airflow resistivities and the high frequency limit tortuosities are still in good compliance with experimental values. The thermal characteristic length of material A has the good order of
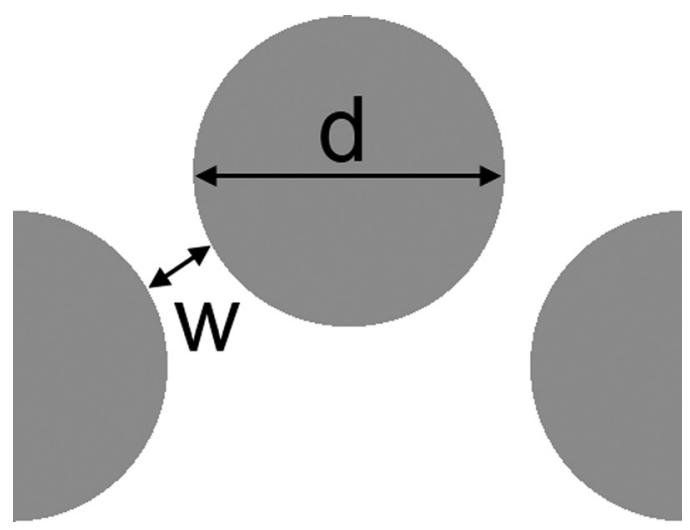

FIG. 7. Two dimensionnal PUC of micro-porous matrix based on a pack of fibers. magnitude. The surprisingly high value of the thermal characteristic length of material $\mathrm{B}$ is due to the predominance of viscous effects which complicates its characterization. When considering a very high resistive material, the determination of thermal parameters $\Lambda^{\prime}$ and $k_{0}^{\prime}$ is less accurate. This explains the very high value of uncertainty of $\Lambda^{\prime}$. This value must be considered with care. At this stage, we remark that the viscous characteristic length is the only parameter that remains overestimated for both materials.

Because viscous boundary layer decreases with frequency, whereas the characteristic cell sizes of the single porosity unit cell remain constants for a given material, inertial transport progressively dominates the whole response-functions at higher frequencies. As the characteristic sizes of the single porosity unit cells remain unchanged, the $\Lambda$ computed coefficient is on the order of the throat radius $R_{t}=D_{t} / 2$ for each foam model. Contrary to simulation results with the single porosity model, in this frequency regime, $\Lambda$ obtained experimentally is much lower than $R_{t}$. Because the computed resistivity (for both materials A and B), which is controlled by the throat size, is in good agreement with measurements, this tends to promote the existence of a second network of smaller interconnecting paths between the main pore sizes, clearly visible on high resolution micrographs (Fig. 2), to be explicitly implemented in the local geometry model.

Adding to the model $n$ secondary small windows of diameter $D_{t s}$ (with a ratio $r=D_{t} / D_{t s}$ ) to the primary windows may decrease the viscous length $\Lambda$ without significant modifications of the resistivity $\sigma$. Indeed the simulations performed on material A, give the resistivity $\sigma$ equal to 88843 N.s. $\mathrm{m}^{-4}(n=4, r=4)$ for a relatively small number of secondary rather large windows, and $90215 \mathrm{~N} \mathrm{~s} \mathrm{~m}^{-4}$ $(n=16, r=6)$ for a relative large number of secondary rather small windows; while the viscous length $\Lambda$ were, respectively, decreasing to 50 and $32 \mu \mathrm{m}$. The viscous lengths were therefore reduced to half of their original values when several secondary window sizes were introduced without significant modifications of the other macroscopic parameters. Thus a simple means to take into account the effect of secondary windows on the overall transport parameters of the unit cell is to modify only $\Lambda$ estimates by considering a parallel network of constant cross section tubes connected to the main pore,

$$
\Lambda_{/ /}=\Lambda \frac{r^{2}+n}{r(r+n)}
$$


where $\Lambda_{/ /}$is an analytic estimate of the viscous length obtained when considering the role of $n$ secondary throat sizes of ratio $r$, and $\Lambda$ is the computed viscous length before the introduction of the secondary throat size network. When applied on single porosity cell, this unit cell is called "single porosity-two throat sizes" in Table I. A further examination of scanning electron micrographs corresponding to material A (Fig. 2) yields the following approximate values: $n=3, r=4$, and $\Lambda_{/ /}=42 \mu \mathrm{m}$. When coupled to double porosity cell, this unit cell is called "double porosity-two throat sizes" and $\Lambda_{/ /}=28 \mu \mathrm{m}$. These values correspond to a secondary window size having typically 5 pixels of diameter. With thus a posteriori evaluation, a resolution of 5 pixels/characteristic diameter was presumably not enough to capture quantitatively the secondary throat size network from x-ray computed microtomography. ${ }^{25}$ This lack of resolution could be overcome using nanotomography instead of microtomography. But some representativeness issues of the corresponding volume would therefore persist. This confirms the interest of our 3D-PUC based method. Note that if an accurate way to quantify this secondary characteristic throat size was available, this would be possible to explicitly introduce it in the model.

These successive improvements directly affect the sound absorption coefficients of material A and B (Figs. 8 and 9, respectively). The "double porosity" cell improves the correlation between simulations and experiments over the entire frequency range because it corrects the porosity for both materials. The consideration of secondary throat size improves the description of the selectivity effect of material A. The consideration of secondary throat size has no effect on material B and is therefore not shown in Table II and Fig. 9.

For the two materials $\mathrm{A}$ and $\mathrm{B}$, we estimated the experimental viscous transition frequencies $f_{v}=\sigma \phi /\left(2 \pi \rho_{0} \alpha_{\infty}\right)$ of 4400 and $24500 \mathrm{~Hz}$ with $\rho_{0}=1.23 \mathrm{~kg} / \mathrm{m}^{3}$, indicating that in the frequency range of interest [0-5000 Hz] the acoustical behavior of material $\mathrm{B}$ is essentially governed by viscous effects, whereas both viscous and inertial effects have to be taken into account to properly model acoustical properties of sample A. This explains why the improvement of the double porosity model compared to the single porosity one in the

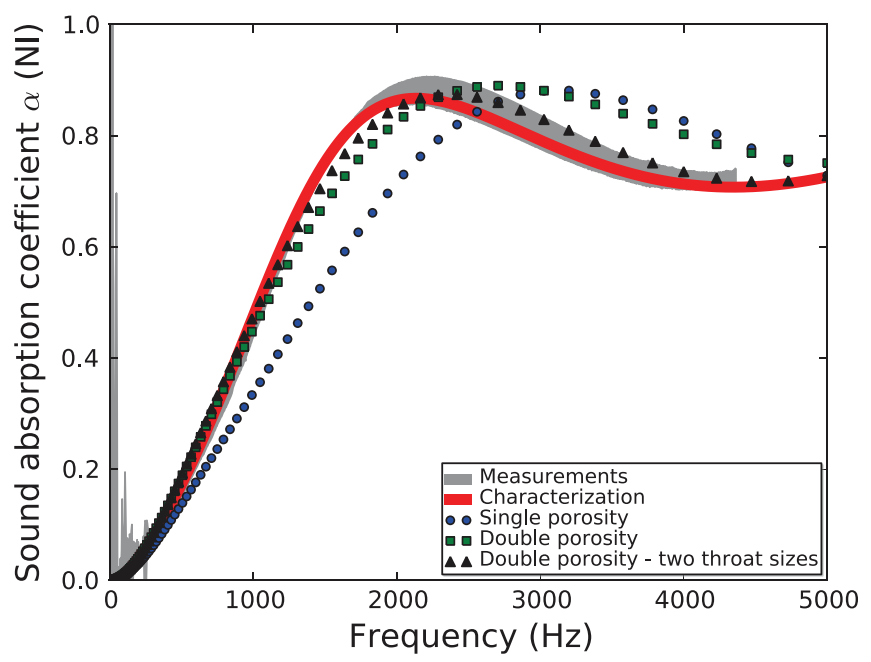

FIG. 8. (Color online) Material A: Comparison of normal incidence (NI) sound absorption coefficient.

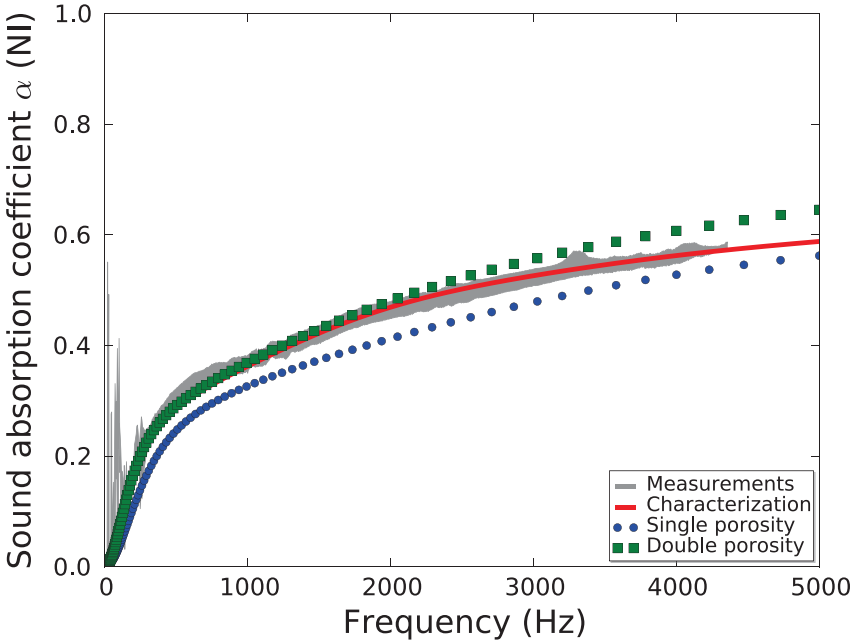

FIG. 9. (Color online) Material B: Comparison of normal incidence (NI) sound absorption coefficient.

prediction of the viscous characteristic length (Table II) does not affect significantly the overall frequency-dependent behavior (Fig. 9): As long as the macroscopic parameters characteristic of the viscous dissipation phenomena are properly predicted by the local geometry model, whether it is a single or a double porosity approach, a good agreement is expected with experiments. Notably, the scaling effect of the porosity which evolves from 0.55 to 0.82 , respectively, for the single- and double porosity cases, is therefore presumably more important for material B than the improvement in modeling the viscous characteristic length provided thanks to a double porosity approach, at least in the frequency range of interest. By contrast, the viscous transition frequency being located in the frequency range of interest, the effect of the viscous characteristic length is strong on the overall response for foam sample A. Therefore each improvement of the local geometry model having a significant influence on the viscous characteristic length prediction is shown to improve substantially the frequency-dependent sound absorption modeling of the foamed material A. As a result, the double porosity-two throat sizes model presented in Fig. 8 exhibits inertial parameters $\alpha_{\infty}$ and $\Lambda$ that are comparable to those of experiments (Table I), leading to values of the sound absorption spectrum that are consistent with impedance tube measurements from low to high frequencies.

\section{Structure-property relations}

This work shows that it is possible to establish a direct link between the microstructure and its acoustical macrobehavior. Particular attention must be paid when choosing and sizing the idealized periodic unit cell. Together with a close match between the macroscopic parameters in the experiments and in the models, this, and the results in the preceding text confirm the accuracy of the numerical models used with the double porosity theory and indicate that they capture the essential physics of the frequency-dependent fluid-structure interactions for a viscous and thermally conducting fluid saturating an air-saturated mineral foam, making it easier to perform parametric and optimization studies in relation with the manufacturing process. 
The secondary throat size allows a finer correlation between modeling and experiments, but the direct link between the microstructure and its acoustical macrobehavior is somewhat broken up because at this time we are not able to perform a statistically representative characterization of these secondary throat size for microtomography resolution reasons.

In ignoring this secondary throat size, a direct link between the microstructure and its acoustical macro-behavior is, however, maintained. Now it is thus possible to discuss with chemists and manufacturers to optimize the cell morphology.

\section{SUMMARY AND CONCLUSIONS}

(1) A unified set of transports and diffusion calculations have been carried out on a family of realistic 3D model porous media. The set of model systems we considered is based on the BCC packing and subsequent overlapping spherical pores. In the packing stage, the throat size between the interconnected spheres was identified from direct measurements on x-ray computed microtomography $(\mu \mathrm{CT})$ images of real mineral foam samples.

(2) For the models studied here, the computed permeability provided an excellent estimate of the measured permeability. Indeed our experience with a number of related models indicates that the throat size controls the permeability of real porous materials. However, the viscous characteristic length $\Lambda$ was overestimated by the model of mono-dispersed spheres.

(3) In considering a second set of smaller pores, our aim was to create a system of secondary throat sizes. Accordingly, the characteristic throat size of this secondary network of interconnections was chosen from scanning electron microscope (SEM) observations (because the $\mu \mathrm{CT}$ resolution was not enough to quantify it properly). Estimates of $\Lambda$ based on this second set of interconnections were more reliable.

(4) We also used double porosity equations in which the micro-porosity of the solid matrix itself was modeled to account for the coupling between the micro- (inter-crystalline porosity) and meso- (bubbles porosity) structures. These equations considerably improved the prediction of all the transport and diffusion-based parameters from which the sound absorption coefficients are determined with good accuracy.

(5) We believe that this choice of multi-scale modeling provides a reasonably accurate representation of the inter-crystalline and pore shapes, clearly predicting the acoustics of two real mineral foam samples, on physical grounds. This leads to suitable models for optimization purposes compatible with manufacturing processes.

\section{ACKNOWLEDGMENTS}

Geert Houvenaghel now at ETEX Dryco is gratefully acknowledged for having led the research project at Lafarge Research Center. We kindly thank Luc Jaouen and Francois-Xavier Bécot from Matelys for the acoustical characterizations and their useful comments. Samuel Meulenyzer is also thanked for his helpful analysis of the $\mathrm{x}$ ray computed microtomographies.

${ }^{1}$ S. Gasser, F. Paun, and Y. Bréchet, "Absorptive properties of rigid porous media: Application to face centered cubic sphere packing," J. Acoust. Soc. Am. 117(4), 2090-2099 (2005).

${ }^{2}$ C. Perrot, F. Chevillotte, and R. Panneton, "Dynamic viscous permeability of an open-cell aluminum foam: Computations versus experiments," J. App. Phys. 103, 024909 (2008).

${ }^{3}$ C. Y. Lee, M. Leamy, and J. Nadler, "Frequency band structure and absorption predictions for multi-periodic acoustic composites," J. Sound Vib. 329(10), 1809-1822 (2010).

${ }^{4} \mathrm{R}$. Venegas and O. Umnova, "Acoustical properties of double porosity granular materials," J. Acoust. Soc. Am. 130(5), 2765-2776 (2011).

${ }^{5}$ C. Perrot, F. Chevillotte, M. T. Hoang, G. Bonnet, F.-X. Bécot, L. Gautron, and A. Duval, "Microstructure, transport, and acoustic properties of open-cell foam samples: Experiments and three-dimensional numerical simulations," J. Appl. Phys. 111, 014911 (2012).

${ }^{6} \mathrm{C}$. Boutin and C. Geindreau, "Estimates and bounds of dynamic permeability of granular media," J. Acoust. Soc. Am. 124(6), 3576-3593 (2008). ${ }^{7} \mathrm{X}$. Olny and C. Boutin, "Acoustic wave propagation in double porosity media," J. Acoust. Soc. Am. 114, 73-89 (2003).

${ }^{8}$ Lafarge Gypsum International, "Acoustic fire-resisting insulating partition, ceiling or lining," European patent application EP 2196589 A1 (June 16, 2010).

${ }^{9}$ P. Spanne, J. F. Thovert, C. J. Jacquin, W. B. Lindquist, K. W. Jones, and P. M. Adler, "Synchrotron computed microtomography of porous media: Topology and transports," Phys. Rev. Lett. 73(14), 2001-2004 (1994).

${ }^{10}$ J. F. Allard and N. Atalla, Propagation of Sound in Porous Media. Modelling Sound Absorbing Materials, 2nd ed. (Wiley, Chichester, UK, 2009), Sec. 5, pp. 73-90 and Sec. 9, pp. 188-198.

${ }^{11}$ D. L. Johnson, J. Koplik, and R. Dashen, "Theory of dynamic permeability and tortuosity in fluid-saturated porous media," J. Fluid Mech. 176, 379-402 (1987).

${ }^{12}$ Y. Champoux and J. F. Allard, "Dynamic tortuosity and bulk modulus in air-saturated porous media,” J. Appl. Phys. 70, 1975-1979 (1991).

${ }^{13}$ D. Lafarge, P. Lemarinier, J. F. Allard, and V. Tarnow, "Dynamic compressibility of air in porous structures at audible frequencies," J. Acoust. Soc. Am. 102, 1995-2006 (1997).

${ }^{14}$ C. Perrot, F. Chevillotte, and R. Panneton, "Bottom-up approach for microstructure optimization of sound absorbing materials," J. Acoust. Soc. Am. 124(2), 940-948 (2008).

${ }^{15}$ D. Weaire and S. Hutzler, The Physics of Foams (Oxford University Press, Oxford, 1999), $264 \mathrm{pp}$.

${ }^{16} \mathrm{~J}$. Serra, Image Analysis and Mathematical Morphology (Academic, New York, 1982), $610 \mathrm{pp}$.

${ }^{17}$ E. Brun, J. Vicente, F. Topin, and R. Occelli, "Geometrical measurement of real foams from 3D images," in Metfoam'07, Montreal, Canada, (September 5-7, 2007).

${ }^{18}$ J.-L. Auriault, C. Boutin, and C. Geindreau, Homogenization of Coupled Phenomena in Heterogeneous Media (Wiley-ISTE, London, UK, 2009), pp. 197-226.

${ }^{19}$ R. J. S. Brown, "Connection between formation factor for electrical resistivity and fluid-solid coupling factor in Biot's equations for acoustic waves in fluid-filled porous media," Geophysics 45, 12-69 (1980).

${ }^{20}$ J. Rubinstein and S. Torquato, "Diffusion-controlled reactions: Mathematical formulation, variational principles, and rigorous bounds," J. Chem. Phys. 88, 63-72 (1988).

${ }^{21}$ L. L. Beranek, "Acoustic impedance of porous materials," J. Acoust. Soc. Am. 13, 248-260 (1942).

${ }^{22} \mathrm{R}$. Panneton and X. Olny, "Acoustical determination of the parameters governing viscous dissipation in porous media," J. Acoust. Soc. Am. 119, 2027-2040 (2006).

${ }^{23} \mathrm{X}$. Olny and R. Panneton, "Acoustical determination of the parameters governing thermal dissipation in porous media," J. Acoust. Soc. Am. 123, 814-824 (2008).

${ }^{24} \mathrm{P}$. Blanc, A. Lassin, and P. Piantone, “Thermoddem a database devoted to waste minerals," Bureau de Recherches Géologiques et Minières (Orléans, France), 2007, http://thermoddem.brgm.fr (Last viewed March 15, 2013).

${ }^{25}$ E. J. Garboczi, M. F. Thorpe, M. S. DeVries, and A. R. Day, "Universal conductivity curve for a plane containing random holes," Phys. Rev. A 43, 64-73 (1991). 